\title{
Effect of Deep Relaxation Technique on Cardiac Autonomic Dysfunction in Type 2 Diabetes Mellitus
}

\author{
Farhana Rahman ${ }^{1}$, Sultana Ferdousi ${ }^{2}$
}

\begin{abstract}
Background: Type 2 Diabetes Mellitus (T2DM) may be associated with autonomic dysfunction. Performance of deep relaxation technique (DRT) may cause upturn of this deranged nerve function. Objective: To evaluate the impact of DRT on cardiac autonomic nerve function by analysis of time domain series of Heart Rate Variability (HRV) in T2DM patients. Methods: This prospective study was carried out on 30 female T2DM patients aged 50-55 years with duration of diabetes of 5-10 years enrolled from the Out Patient Department of Endocrinology, Bangabandhu Sheikh Mujib Medical University, Dhaka. They performed DRT (20 minutes twice daily) for 3 months. Thirty apparently healthy female with similar age who did not perform DRT or any other form of exercise, were included as control. To assess the cardiac autonomic nerve function, time domain measures of HRV of all subjects were recorded by a data acquisition device Power Lab (Australia). HRV data of all subjects were collected at baseline (pre) and also after 3 months(Post). For statistical analysis, paired and independent sample t-test were used. Results: The pre-intervention values of resting HR was significantly higher $(\mathrm{p}<0.001)$ and mean R-R interval, SDRR, CVRR, SDSD, RMSSD and pRR50\% were significantly lower $(\mathrm{p}<0.001)$ in all diabetic patients compared to control. After 3 months of DRT, there was significant decrement in resting mean heart rate $(\mathrm{p}<0.01)$ and significant increment in mean $\mathrm{R}-\mathrm{R}$ interval, SDRR, CVRR, SDSD, RMSSD and pRR50\% $(\mathrm{p}<0.001)$ compared to their pre-intervention values. Conclusion: Cardiac autonomic nerve dysfunction may occur in T2DM and 3 months regular practice of DRT mayy significantly improv cardiac autonomic nerve function with parasympathetic dominance.
\end{abstract}

Keywords: Deep relaxation technique, Autonomic nerve function, Heart rate variability, type 2 Diabetes mellitus.

J Bangladesh Soc Physiol. 2018, June; 13(1): 22-28

For Authors Affiliation, see end of text.

http://www.banglajol.info/index.php/JBSP

DOI: http://dx.doi.org/10.3329/jbsp.v13i1.37845

\section{Introduction}

7 ype 2 diabetes mellitus (T2DM) is the most widely recognized type of diabetes. ${ }^{1}$ Cardiac autonomic neuropathy (CAN) is the most prominent focus of diabetes because of the life-threatening consequences of this complication. ${ }^{2} \mathrm{CAN}$ may

Received 09 Jan. 2018; $\quad$ Accepted 15 April 2018 be clinically apparent or in apparent. ${ }^{3}$ Subclinical CAN does not manifest any symptoms of CAN and clinical CAN may be manifested by resting tachycardia, orthostatic hypotension, exercise intolerance and silent mayocardial ischemia. ${ }^{4}$ Once the feature of CAN is clinically evident, it cannot be reversed effectively by any treatment. ${ }^{5}$ Therefore, it is plausible to intervene at its subclinical stage in diabetic patients. 
In recent days, various relaxation techniques demonstrated promise to help to cope with various stress related health problems to such as-cancer and pain. ${ }^{6}$ Significant improvement in angina, diabetes, cardiac arrhythmias, hypertension, bronchial asthma, insomnia, rheumatoid arthritis has been reported with trial of these yoga based relaxation therapy. ${ }^{7}$ Among relaxation techniques, deep relaxation technique (DRT), cyclic meditation (CM), Shavasan and different form of meditation have been studied by several investigators. ${ }^{8-10}$ In addition, DRT has been ascribed as a procedure of meditation in which the subject adopts a posture comfortable for him/her usually in supine posture with eyes closed. Then he/she focuses mind on breathing which leads to the state of relaxation of body and mind. ${ }^{7,9}$

Assessment of HRV has been recently recognized as a tool to predict subclinical CAN before the disease is symptomatic. ${ }^{3,11}$ It is non invasive, accurate and generally reproducible technique. ${ }^{12}$ Mean heart rate (HR), mean R-R interval, standard deviation of RR interval (SDRR), the coefficient of variation of the RR interval (CVRR), the standard deviation of the differences of successive RR intervals (SDSD), square root of mean squared differences of successive RR intervals (RMSSD) and the proportion derived by dividing number of RR interval $>50 \mathrm{~ms}$ by the total number of RR interval (pRR50\%) are considered as time domain measures for HRV measurement in which mean HR, SDRR are the net interaction of both sympathetic and parasympathetic nerve function activity and mean R-R interval, CVRR, RMSSD, SDSD and pRR50\% reflect cardiac parasympathetic modulation. ${ }^{13-18}$ Type 2 diabetic patients has been found associated with decrement of cardiac parasympathetic activity. ${ }^{12,13-15,19-20}$
There are reports of improvement of autonomic nervous activities as a result of regular practice of relaxation technique in healthy subjects. ${ }^{21-26}$ Significant increment of SDRR, mean R-R interval, RMSSD, pRR50\% in healthy volunteers after practicing relaxation technique has been reported and significant reduction in resting $\mathrm{HR}$ in healthy volunteers as well as in ischemic heart disease. ${ }^{21-}$ ${ }^{26}$ On the contrary, no change in mean HR, SDRR, RMSSD and $\mathrm{pRR} 50 \%$ after practicing relaxation technique for 3-15 years in healthy volunteers and for 1 week in T2DM was also reported. ${ }^{27-28}$

Though, the benefit of relaxation technique on autonomic function has been tested in healthy subjects, ${ }^{21,23-27}$ the information about the effect of relaxation technique in diabetic patients for it autonomic benefit is lacking and the effect of deep relaxation technique on HRV in sedentary female was investigated ${ }^{26}$ but the data on effect of deep relaxation technique on HRV in T2DM has not been published yet. Therefore, this study has been designed to evaluate the impact of DRT on autonomic tone in patients with T2DM so that the outcome can be used to suggest ii as an adjunct to typical treatment strategy for T2DM to improve the cardiac autonomic dysfunction and also to reduce cardiovascular \& cardiac autonomic neuropathy, morbidities, long term complications and ensure healthy life in diabetic patients.

\section{Methods}

This prospective study was conducted during 2016 in the Department of Physiology, Bangabandhu Sheikh Mujib Medical University, Dhaka. The protocol of this study was approved by the institutional review board of BSMMU. This study enrolled 30 diagnosed female patients of T2DM following criteria of $\mathrm{WHO}^{29}$ (age: 50 to 55 years; $\mathrm{HbA} 1 \mathrm{C}: 5-10 \%$; 5 to 10 years duration of DM) by consecutive sampling from the Endocrinology Out Patient Department, BSMMU, 
after taking written informed consent. All these patients were only under oral hypoglycemic agents in addition to lifestyle modification. They were studied at baseline before beginning DRT and same patients were studied after performing DRT for 3 months. Age and physical activity matched 30 apparently healthy female previously never exposed to relaxation technique were recruited as control among the relatives and attendants of patients, hospital staffs and also through personal contacts. HRV data of all patients were recorded once before DRT then once after completion of 3 months of DRT. Similarly, data of healthy subjects were recorded at baseline and after 3 month. History of DAN, T1DM, diabetic retinopathy, nephropathy, hypertension, coronary artery diseases, epilepsy, migraine, psychiatric disorders, respiratory disorders, hypo \& hyperthyroidism, consuming drugs with effect on autonomic nervous system function, yoga practitioners and athletes were excluded. The aim and objectives of the study were explained and they were encouraged for voluntary participation. Subsequently, a thorough clinical examination was done and a detail family and medical histories and also physical activity status were recorded in a prefixed data schedule.

For HRV recording, the finally selected subjects were prepared from one day prior to the test. They were advised to take their meal by $9.00 \mathrm{p} . \mathrm{m}$. and have sound sleep and avoid any physical or mental stress and also sedative medications. In addition, they were advised to take light breakfast in the morning without tea and coffee and to attend at the Autonomic Nerve Function Test Laboratory in the Department of Physiology of BSMMU between 9.00 a.m. to 11.00 a.m. on the day of examination. For HRV measurement, the room temperature of the Autonomic Lab was maintained around $25^{\circ} \mathrm{c}-28^{\circ} \mathrm{c}$ and the subject was allowed to sit for 15 minutes to adjust with the lab conditions. Then, ECG was recorded on Lead
II for 5 minutes, by Power Lab 8/35 (ADInstrument, Australia) from which measures of HRV was analysed by Lab chart software.

The patients were asked to perform DRT twice daily for 3 months. For this purpose, a training session was provided to group of selected patients where the researcher demonstrated the procedure by acting DRT for twenty minutes to patients and then supervise their performance and ensure that they can act independently at home. During this session, the participants laid in supine position with eyes closed, then each specific part of the body were relaxed sequentially from tip of the toes to the neck with chanting "AU-M". ${ }^{10,21}$ They were requested to practice the steps twice daily in peaceful, lighted and well ventilated room at home.

Data were expressed as mean and SE. SPSS for windows, version 22.0 was used for data analysis. Independent sample t-test was done to compare the mean values between control and T2DM at their baseline and after 3 months and also paired sample t-test was done to compare mean values of data between before and after intervention with DRT. $p$ value of $<0.05$ was taken as statistically significant.

\section{Results}

In this study, the baseline resting pulse rate, SBP, DBP, and mean HR were found significantly higher $(p<0.01, p<0.001)$ whereas mean R-R interval, SDRR, CVRR, SDSD, RMSSD and pRR $50 \%$ were found significantly lower $(p<0.001)$ in T2DM compared to non diabetic control subjects (Table I and II). Again, after 3 months of DRT, the mean values of SBP, DBP, mean HR, mean R-R interval, SDRR, SDSD and RMSSD were significantly improved $(p<0.01, p<0.001)$ in T2DM and found close to that of non diabetic control subjects. Although values of CVRR, pRR $50 \%$ had been improved in diabetic patients after DRT but these were not similar to control subjects (Table I and II). 
Table I: General characteristics in different groups $(\mathrm{n}=90)$

\begin{tabular}{lcclccc}
\hline Parameters & \multicolumn{2}{c}{ Control $(\mathrm{n}=30)$} & & \multicolumn{2}{c}{ T2DM $(\mathrm{n}=60)$} & Change \\
\cline { 2 - 3 } & Baseline & after 3 months & & Baseline & after 3 months & $\%$ \\
\hline Pulse (bpm) & $81.23 \pm 1.05$ & $74.61 \pm 0.26$ & & $86.50 \pm 0.86^{* *}$ & $75.76 \pm 0.71^{\$ \$}$ & $-12.42 \%$ \\
SBP(mm of hg) & $124.84 \pm 1.19$ & $121.61 \pm 1.30$ & & $130.88 \pm 1.14^{* *}$ & $130.96 \pm 1.33^{\$}$ & $-05.84 \%$ \\
DBP(mm of hg) & $76.35 \pm 0.85$ & $72.97 \pm 0.79$ & & $83.97 \pm 1.00^{* *}$ & $84.61 \pm 0.10^{\$ \$}$ & $-10.86 \%$ \\
\hline
\end{tabular}

Data were expressed as mean \pm SE. Statistical analysis was done by independent sample t-test and paired sample t-test. $\mathrm{SBP}=$ systolic blood pressure; $\mathrm{DBP}=$ diastolic blood pressure; $(*=$ control baseline vs T2DM baseline; $\$=$ T2DM baseline vs T2DM after 3 months; $* *=p<0.001 ; \$=p<0.01 ; \$ \$=p<0.001$; $\mathrm{n}=$ number of subjects.)

Table II: Time domain measures of HRV in different groups ( $\mathrm{n}=90)$

\begin{tabular}{lcccccc}
\hline Parameters & \multicolumn{2}{c}{ Control $(\mathrm{n}=30)$} & & \multicolumn{2}{c}{ T2D M(n=60) } & Change \\
\cline { 2 - 3 } & Baseline & after 3 months & & Baseline & after 3 months & $\%$ \\
\hline Mean HR (bpm) & $73.05 \pm 0.87$ & $70.33 \pm 0.97$ & & $84.31 \pm 1.61^{* *}$ & $74.81 \pm 0.83^{\$}$ & $-11.26 \%$ \\
R-R interval(sec) & $0.83 \pm 0.00$ & $0.84 \pm 0.01$ & & $0.71 \pm 0.01^{* *}$ & $0.83 \pm 0.01^{\$}$ & $+16.60 \%$ \\
SDRR(ms) & $44.20 \pm 1.58$ & $42 \pm 1.29$ & & $19.22 \pm 1.37^{* *}$ & $40.17 \pm 1.53^{\$ \$}$ & $+109 \%$ \\
CVRR & $0.05 \pm 0.00$ & $0.05 \pm 0.00$ & & $0.02 \pm 0.00^{* *}$ & $0.04 \pm 0.00^{\# \$}$ & $+80.77 \%$ \\
SDSD (ms) & $31.69 \pm 1.88$ & $34.01 \pm 1.91$ & & $10.73 \pm 0.99^{* *}$ & $30.60 \pm 1.80^{\$ \$}$ & $+185.18 \%$ \\
RMSSD (ms) & $31.65 \pm 1.88$ & $34.00 \pm 1.92$ & & $10.72 \pm 0.99^{* *}$ & $30.59 \pm 1.80^{\$ \$}$ & $+185.35 \%$ \\
pRR50\% & $11.84 \pm 1.77$ & $13.61 \pm 0.02$ & & $0.51 \pm 0.00^{* *}$ & $5.03 \pm 0.01^{\# \$}$ & $+886.27 \%$ \\
\hline
\end{tabular}

Data were expressed as mean \pm SE. Statistical analysis was done by independent sample t-test and paired sample t-test. $S D R R=$ Standard deviation of $R R$ interval; $C V R R=$ Coefficient of variation of the $\mathrm{RR}$ intervals; $\mathrm{SDSD}=$ Standard deviation of differences between successive RR interval; RMSSD= Square root of mean of squared differences of successive RR interval ; $p R R 50 \%=$ Number of RR interval differing by $>50 \mathrm{~ms}$ from adjacent intervals divided by the total number of RR intervals $(*=$ control baseline vs T2DM baseline; \#= control after 3 months vs T2DM after 3 months; $\$=$ T2DM baseline vs T2DM after 3 months; $* *=p<0.001 ; \#=p<0.001 ; \$=p<0.01 ; \$ \$=p<0.001 \mathrm{n}=$ number of subjects.)

\section{Discussion}

In the present study significantly higher values of mean HR and significantly lower values of mean R-R interval, SDRR, CVRR, SDSD, RMSSD and $\mathrm{pRR} 50 \%$ suggest higher sympathetic tonic discharge and lower cardiac vagal tone in all diabetic patients before intervention were almost similar to the results of other investigators. ${ }^{14,19,30-}$ ${ }^{34} \mathrm{It}$ is interesting to note that after 3 months of regular practice of DRT the diabetic patients showed significant improvement of autonomic nerve function supported by significant decrease in mean HR (11.26\%) and significant increase in mean R-R interval (16.60\%), SDRR (109\%), 
CVRR(80.77\%), SDSD (185.18\%), RMSSD $(185.35 \%)$ and $\mathrm{pRR} 50 \%(886.27 \%)$. These results were consistent with other studies conducted to observe the effect of DRT on cardiac autonomic nerve function but they observed in healthy subjects. $^{21,23-26}$

In addition, DRT also showed very good improvement in some other measures of parasympathetic tone by substantial increase in post intervention data of CVRR, SDSD and RMSSD which further intensifies the evidence of improvement of cardiac parasympathetic tone in T2DM.

To summarize, results of this prospective study showed the impaired autonomic nerve function activity in T2DM patients and reversal of this autonomic nerve dysfunction was evident by regular practice of DRT for 3 months causing markedly increased parasympathetic activity almost similar to that in healthy non diabetic people.

The exact mechanism involved in the autonomic response to this kind of yogic relaxation response has not been clearly delinated. But the body of literature review proposed, the highly focused attention and willful muscle relaxation which is a part of the procedure of DRT probably created an integrated hypothalamic response by neural signal from increase activation of pre frontal cortex (PFC), anterior cingulated cortex (ACC) and other part of limbic system with a consequence of parasympathetic predominance in the diabetic patients. ${ }^{7,9,35-43}$ From the above feature it is apparent that DRT have been found to exert profound effect on restoration of autonomic balance to reduce cardiovascular \& cardiac autonomic neuropathy, morbidity and thus ensure healthy life in type 2 diabetic patients.

\section{Conclusion}

From this study it can be concluded that there was dysautonomia characterized by depressed parasympathetic in T2DM which was counter balanced by exercising DRT for 3 months.
Therefore, it is evident that regular performance of deep relaxation technique is an effective measure to improve autonomic nerve dysfunction in its subclinical stage and can delay and prevent clinical autonomic neuropathy in type 2 diabetic mellitus.

\section{Acknowledgement}

Authors of this study are thankful to the authority of the Department of Endocrinology, BSMMU for their cooperation during this study.

\section{Conflict of interest None}

Author affiliations

*1. Farhana Rahman, Assistant Professor, Department of Physiology, Sir Salimullah Medical college, Dhaka. Email: rum121285@gmail.com.

2. Sultana Ferdousi, Professor, Department of Physiology, Bangabandhu Sheikh Mujib Medical University (BSMMU), Bangladesh. Email: sferdousiratna@gmail.com._sferdousiratna@ bsmmu.edu.bd

*For correspondence

\section{References}

1. Alberti MMDK and Zimmet ZP . Diagnosis and classifictaion of diabetes mellitus, provisional report and classification. Dia Med. 1998;15: 539-53.

2. Boulton AJM, Vinik AI, Arezzo JC, Bril V, Feldman LE, Freeman R, Mallik RA, Maser RE, Sosenko JM Ziegler D. Diabetic neuropathies. Diabetes Care. 2005; 28(4): $956-62$

3. Dimitropoulos G, Tahrani AA, Stevens MJ. Cardiac autonomic neuropathy in patients with diabetes mellitus. World J Diabetes.2014; 5(1): 17-39.

4. Vinik AI, Maser RE, Mitchell BD, Freeman R. Diabetic Autonomic Neuropathy. Diabetes Care. 2003; 26 (5): 1553-79.

5. Verrotti A, Prezioso G, Scattoni R, Chiarelli F. Autonomic neuropathy in diabetes mellitus. Front. Endocrinol. 2014; 5: 1-5.

6. Relaxation techniques: try these steps to reduce stress. [Internet].[cited 2016 Oct 3]. Available from: Mayoclinic.com.

7. Benson H, Klipper MZ. The Relaxation Response. $1^{\text {st }}$ ed. USA: Harper Collins; 1975.

8. Amita S, Prabhakar S, Manoj I, Harminder S, Pavan T. Effect of yoga-nidra on blood glucose level in diabetic patients. Indian J Physiol Pharmacol. 2009; 53(1): 97-101.

J Bangladesh Soc Physiol. 2018, June; 13(1): 22-28 
9. Cohen DL, Wintering N, Tolles V, Townsend RR, Farrar JT, Galantino ML, Newberg AB. Cerebral blood flow effects of yoga training: Preliminary evaluation of 4 cases. Alter Comple Medi. 2009; 15(1): 9-14.

10. Khemka SS, Rao NH and Nagarathna R. Immediate effects of two relaxation techniques on healthy volunteers. Ind J Phy Phar. 2009; 53: 67-72.

11. Balcioglu S, Arslan U, Turkoglu S, Ozdemir M, Cengel A. Heart rate variability and heart rate turbulence in patients with Type 2 diabetes mellitus with versus without cardiac autonomic neuropathy. 2007; doi: 10.1016/j.amjcard.2007.03.106.

12. Al-Hazimi A, Al-Ama, Syiamic Ahmad, Qosti R, and Abdel-Galil $\mathrm{K}$. Time-domain analysis of heart rate variability in diabetic patients with and without autonomic neuropathy. Annals S Med. 2002; 22 (5-6): 400-403.

13. Task Force of the European Society of Cardiology and the North American Society of pacing and electrophysiology. Heart Rate Variability: Standards of Measurement, Physiological Interpretation and Clinical Use. Circulation. 1996; 93: 1043- 65.

14. Goit RK, Paudel BH, Sharma SK, Khadka R. Heart rate variability and vibration perception threshold in type 2 diabetes mellitus. Int J Diabet Dev Ctries. 2013; 33(3): 134-9.

15. Pramodh V, Kumar MP and Prasad BAK. Heart rate variability in type 2 diabetics. IJBR. 2014; 5(5): 364-7.

16. Dekker JM, Crow RS, Folsom AR, Hannan PJ, Liao D, Swenne CA, Schouten EG. Low heart rate variability in a 2-minute rhythm strip predicts risk of coronary heart disease and mortality from several causes. Circulation. 2000;102:1239-44.

17. Shimazaki M, Kikuchi K, Kobayakawa H, Yamamoto M, Kudoh C, Wada A, Sakamoto T, Sawai N, Mukai H, Limura O . The coefficient of variation of $R R$ intervals (CVRR) on electrocardiogram in patients with essential hypertension with reference to aging, hemodynamics and sympatho-adrenomedullary function. Jpn J Geriatrics. 1991; 28(5): 640-5.

18. Yadav KPS and Saini BS. Study of the aging effects on HRV measures in healthy subjects. Int J Comput Theo Eng. 2012; 4(3):346-9.

19. Kudat H, Akkaya V, Sozen AB, Salman S, Demirel S, Ozcan M, Atilgan D, Yilmaz MT, Guven O. Heart
Rate Variability in Diabetes Patients. J Int Med Res. 2006; 34: 291-6.

20. Yokoayama A. Progostic significance of QT prolongation and autonomic nervous dysfunction in alcoholics with diabetes mellitus. Keio J Med. 1993;42(4): 141-8.

21. Vempati RP and Telles S. Yoga based guided relaxation reduces sympathetic activity in subjects based on baseline levels. Psychol Rep.2002; 90(2): 487-94.

22. Dixhoorn JV, White A. Relaxation Therapy for Rehabilitation and Prevention in Ischemic Heart Disease: A systematic review and meta analysis. Eur J Cardiovasc Prev Rehabil. 2005; 12:193-202.

23. An H, Kulkarni R, Nagarathna R, Nagendra HR. Measures of HRV in women following a meditation technique. Int J Yoga. 2010; 3(1): 6-9.

24. Patra $\mathrm{S}$ and Telles $\mathrm{S}$. Heart rate variability during sleep following the practice of cyclic meditation and supine rest. Appl Psy Physiol and Biofeedback. 2010; 35(2):135-40.

25. Telles S, Raghavendra BR, Naveen KV, Manjunath NK, Kumar S, Subramanya P. Changes in autonomic variables following two meditative states described in yoga texts. J Alter Compli Medi. 2013; 19(1):3542 .

26. Fatema ME, Begum $\mathrm{N}$ and Ferdousi S. Effect of deep relaxation on heart rate variability in sedentary females. J Bangladesh Soc Physiol. 2013; 8(2): 6559.

27. Peng CK, Henry IC, Mietus JE, Hausdorff JM, Khalsa G, Benson H, Goldberger AL. Heart rate dynamics during three forms of meditation. Int $\mathrm{J}$ of Cardiol. 2004; 95: 19-27.doi:10.1016/ ijcard.2003.02.006.

28. Vinutha HT, Raghvendra BR and Manjunath NK . Effect of Integrated Approach of Yoga Therapy on Autonomic Functions in Patients with Type 2 Diabetes.Indian J Endo Metab. 2015;19(5):653-7.

29. World Health Organization. Definition and diagnosis of diabetes mellitus and intermediate hyperglycemia: Report of a WHO/IDF Consultation. [Internet]. Geneva: World Health Organization; c2006. [Cited 2016 Mar 13]. Available from: https://www.idf.org.

30. Malpas SC and Maling TJB . Heart-Rate Variability and Cardiac Autonomic Function in Diabetes. Diabetes. 1990;39: 1177-81. 
31. Kataoka M, Ito C, Sasaki H, Yamane K, Khono N . Low heart rate variability is a risk factor for sudden cardiac death in type 2 diabetes. Diabetes Res Clin Pract. 2004; 64: 51-58.

32. Syed PTA, Ahmed VIT, Jacob J, Joseph P. Time and frequency domain analysis of heart rate variability and their correlations in diabetes mellitus. World Academy of Science, Engineering and Technology. 2008; 15: 624-627.

33. Tarvainen MP, Laitinen TP, Lipponen JA, Cornforth DJ, Jelinek HF. Cardiac autonomic dysfunction in type 2 diabetes - effect of hyperglycemia and disease duration. Front Endocrinol. 2014; 5: 1-9.

34. Phurpa. Analysis of heart rate variability in Type 2 diabetes mellitus [Thesis][Dhaka]: Bangabandhu Sheikh Mujib Medical University; 2016.

35. Tang YY, Ma Y, Fan Y, Feng H, Wang J, Feng S, Lu Q, Hu B, Lin Y, Li J, Zhang Y, Wang Y, Zhou L, Fan M. Central and autonomic nervous system interaction is altered by short-term meditation. PNAS. 2009; 106(22):8865-70.

36. Dooley C. The impact of meditative practices on Physiology and Neurology: A review of the literature. Scientia Discipulorum. 2009; 4: 35-59.

37. Mills PG, Schneider RH, Hill D, Walton KG, Wallace RK. Beta-adrenergic receptor sensitivity in subjects practicing transcendental meditation. J Psychosom
Res. 1990; 34(1): 29-33.doi.10.1016/00223999(90)90005-O.

38. Matthews SC, Paulus MP, Simmons AN, Nelesen RA, Dimsdale JE. Functional subdivisions within anterior cingulate cortex and their relationship to autonomic nervous system function. Neuroimage.2004; 22: 1151-6.

39. Lazar SW, Bush G, Gollub RL, Fricchione GL, Khalsa G, Benson H. Functional brain mapping of the relaxation response and meditation. Neuro Report. $2000 ; 11: 1581-5$

40. Newberg AB, Alavi A, Baime MJ, Pourdehnad M, Santanna J, D'Aquili E. The measurement of regional cerebral blood flow during the complex cognitive task of meditation: a preliminary SPECT study. Psychia Res. 2001; 106(2): 113-22.

41. Conrad A and Roth WT. Muscle relaxation therapy for anxiety disorders: it works but how? Anxiety Disorder. 2007; 21: 243-64.

42. Davidson RJ, Kabat-Zinn J, Schumacher J, Rosenkranz M, Muller D, Santorelli SF, Urbanowski F, Harrington A, Bonus K, Sheridan JF. Alterations in brain and immune function produced by mindfulness meditation. Psychosom Med 2003; 65: 564-70.

43. Keily WF, Gellhorn E. Mystical states of conciousness: Neurophysiological and clinical aspects. J Nerv Mental Dis. 1972; 154(6): 399405 . 